\title{
Papers
}

\section{Laboratory facilities for investigating lipid disorders in the United Kingdom: Results of the British Hyperlipidaemia Association Survey}

\author{
M F Laker, J P D Reckless, D J Betteridge, P N Durrington, J P Miller, D P Nicholls, \\ J Shepherd, G R Thompson
}

\begin{abstract}
Aims: To determine the availability of facilities for the investigation of hyperlipidaemia in the United Kingdom.

Methods: A questionnaire was sent to all health districts in the United Kingdom.

Results: The response rate was $81 \%$. All laboratories used enzymatic techniques to measure serum triglyceride and cholesterol concentrations, although there were differences in standardisation procedures. Reference ranges for serum lipids were quoted by $58 \%$ of laboratories while $50 \%$ quoted "desirable limits". Almost half specified that fasting blood samples were required. High density lipoprotein cholesterol concentrations were estimated by $75 \%$ and apolipoproteins $A I$ and $B$ by $14 \%$ of laboratories; there were differences in specimen type and considerable diversity in procedures used for measurement.
\end{abstract}

Conclusions: Many laboratories were unaware of current recommendations for screening for hypercholesterolaemia in the community. The present survey indicated an urgent need for the introduction of better reference methods, standardisation, and quality assurance procedures before apolipoproteins become a routine part of coronary heart disease risk assessment.

The detection and management of patients with disorders of lipoprotein metabolism are provoking considerable interest. This is because clinical trial results have shown that lowering serum cholesterol reduces coronary heart disease morbidity ${ }^{12}$ and angiographic evidence that effective treatment of hyperlipidaemia may limit the progression or induce regression of atherosclerosis. ${ }^{3-5}$ As a result several sets of consensus guidelines for desirable limits for blood lipid concentrations and the treatment by which these may be achieved have been published. ${ }^{6-9}$

These recommendations make two assumptions:

1 That clinical services have been developed which specialise in the management of patients whose hyperlipidaemia is refractory to measures initiated in general practice. Clinical provision in this area in the United Kingdom, however, seems to have occurred largely on an ad hoc basis. There is no recognised subspecialty of "lipidology," and physicians with an interest in lipid disorders have a variety of training backgrounds. Until recently no appropriate formal training scheme had been recognised by the Royal Colleges.

2 Interlaboratory factors affecting lipid measurement, such as inaccuracy and bias, are minimal, but there is good evidence to suggest that this is not true. ${ }^{10}$ Because national or international consensus values of serum lipid concentrations are used for management decisions, locally determined reference intervals will not compensate for method dependent bias.

The British Hyperlipidaemia Association (BHA) therefore undertook a national survey of current clinical and laboratory practice for the investigation and management of patients with hyperlipidaemia. Additional objectives were to establish registers of lipid clinics and laboratories which carry out tests that are not generally available but which might be required for the investigation of patients with rare abnormalities. The results on clinic practice, published elsewhere, ${ }^{11}$ showed that physicians involved in lipid clinics came from a variety of specialty backgrounds, chemical pathology ( $43 \%)$ providing the largest number. Our report presents findings on the availability of laboratory facilities.

\section{Methods}

A questionnaire was prepared which explored clinical facilities and the laboratory support available for the investigation and management of patients with hyperlipidaemia. This was sent to the chairmen of the divisions of medicine in all 202 health districts and special hospital authorities in England and Wales, and the 21 health boards in Scotland, Northern Ireland, and the Channel Islands. Copies of the questionnaire were also sent to all hospitals in the United Kingdom with more than 100 beds and to BHA members, to elicit a response from as many districts as possible. Recipients were asked to pass the questionnaire to the local clinician most closely involved in the management of hyperlipidaemic patients and then to the laboratory for completion.

The questionnaire was circulated in July 1988. The data from completed questionnaires were entered into a computer using dBase III plus (Ashton-Tate, Maidenhead, Berks) and 
Figure 1 Methods of calibrating serum lipid measurements. Serum: serum based calibrators; CDC: serum based calibrators which were traceable to Centers for Disease Control material, Calibration: calibration based on extinction coefficients.
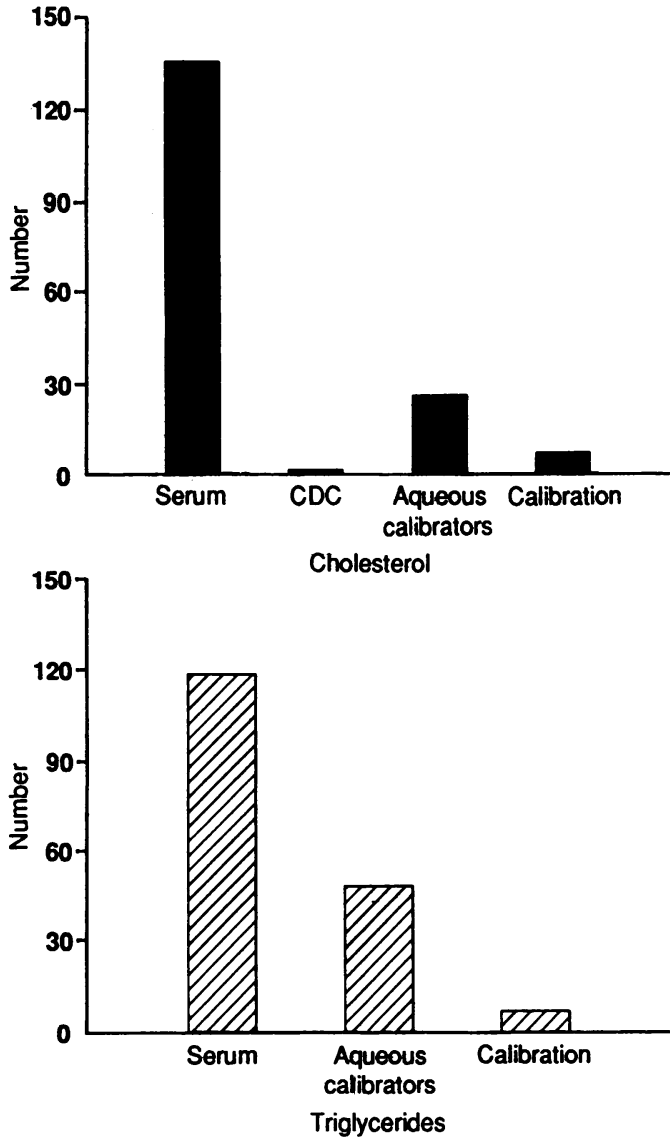

analysed using Statgraphics (STSC Inc, 2115 East Jefferson Street, Rockville, Maryland, USA). To ensure that as many facilities as possible were included the results of the computer analysis were cross-checked by BHA committee members for their regions and with the entries for Northern Ireland in the Handbook of the Irish Hyperlipidaemia Association. A single entry was made where duplicate responses were received. Preliminary data were presented at the BHA annual scientific meeting in June 1989 and arising from this some further responses and corrections were received. These were included in the final analysis.

\section{Results}

LIPID AND LIPOPROTEIN ASSAYS

There were $188(81 \%)$ responses to the laboratory questionnaire, although not all sections were completed. All laboratories that responded provided cholesterol measurements using enzymatic techniques, and all but two laboratories offered triglyceride measurement, also by enzymatic methods. Most laboratories used serum based material to calibrate methods for lipid measurement, although procedures based on aqueous calibrators or calculation using extinction coefficients were used by $20 \%$ for cholesterol and $31 \%$ for triglycerides (fig 1). In all instances, total lipids were determined using serum rather than plasma, 83 departments specifying fasting blood samples, while 56 accepted non-fasting blood samples, and 31 specified fasting specimens if investigations included triglyceride estimation, but accepting non-fasting specimens if cholesterol only was requested. Lipoprotein electrophoresis was undertaken by $79(42 \%)$ laboratories, the service being restricted to patients with suspected type III (remnant) hyperlipoproteinaemia in 12 , raised serum triglyceride concentrations in 10, and combined hyperlipidaemia in 16 departments. Lipoprotein fractionation and quantitation using ultracentrifugation was available in 12 departments.

The measurement of high density lipoprotein (HDL) cholesterol was available from $136(72 \%)$ laboratories of which $94(70 \%$ of laboratories measuring HDL cholesterol) provided a service limited either to a special arrangement or to specialised clinics, particularly for control of lipids or diabetes. All but 17 laboratories used serum specimens, seven used anticoagulated (heparin) plasma, and 10 EDTA plasma. Ultracentrifugation was used to isolate HDL in one laboratory, the others using methods depending on the precipitation of apolipoprotein $\mathbf{B}$ containing lipoproteins. The precipitants used are shown in fig 2 . All respondents participated in at least two external quality assurance schemes (EQAS) for cholesterol and triglyceride measurements, but there is no such scheme presently available for HDL cholesterol determination, although two regions ran local schemes.

Reference ranges for serum lipid concentrations were quoted by $65(35 \%)$ respondents, "desirable limits" by $51(27 \%)$, both by 43 $(23 \%)$, and six $(3 \%)$ provided no reference data. There were no responses from $23(12 \%)$.

\section{APOLIPOPROTEIN AND ENZYME ASSAYS}

Apolipoprotein (apo) AI and B measurements were available in 26 laboratories, apo CII detection in three, apo. E phenotyping in 12, lipoprotein (a) in eight, lipoprotein lipase activity (or post-heparin lipolytic activity) in seven. A list of laboratories that are prepared to offer these and some additional services to other departments, together with conditions for accepting specimens, will be provided on request (MFL). Methods used for apolipoprotein determinations were radial immunodiffusion (8), electroimmunoassay (4), radioimmunoassay (3) and immunoturbidometric procedures (20). Apolipoprotein assays were

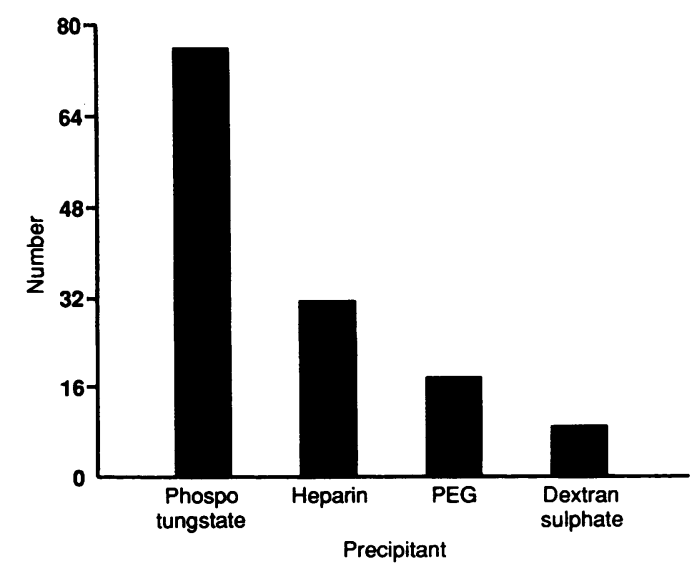

Figure 2 Precipitants used in HDL cholesterol measurement. 
standardised using a variety of commercial material. There is no national EQAS for apolipoproteins.

\section{Discussion}

Findings from a questionnaire for which a response rate of $81 \%$ was obtained may be open to bias as it is likely that those most interested in the subject of the questionnaire will have responded. The response rate was high, however, and it seems likely that our data are representative of practice in the United Kingdom.

All laboratories used enzymatic techniques for measuring serum cholesterol and triglyceride concentrations using a variety of analysers; no laboratory was using Liebermann-Burchard colorimetric methods. Improvements in interlaboratory comparability of cholesterol analyses have been noted in recent years, attributed, in part, to the use of more specific enzyme reagents and improved automated analysers. Imprecision, however, still varies between 2.2 and $13.7 \%$ with these techniques. ${ }^{10}$ The present survey shows that there is considerable diversity in calibration procedures. Improvements in analytic performance have been reported when serum calibrators are used with values assigned using the US Centers for Disease Control (CDC) modification of the Abell-Kendall procedure for estimating cholesterol. ${ }^{12}$ Minimisation of bias (inaccuracy) and interassay imprecision values of less than $3 \%$ should be the aim for tests which should be interpreted on the basis of risk of coronary heart disease rather than by comparison with reference values from local populations in which high serum cholesterol concentrations are prevalent. ${ }^{6-9}$ Many laboratories $(35 \%)$ are still using outdated high reference ranges for serum cholesterol concentrations.

It is particularly noteworthy in the light of recent recommendations with regard to screening for hypercholesterolaemia that $49 \%$ of laboratories still insisted on fasting blood samples. This is a major obstacle to screening in the community, being an unnecessary restraint because fasting makes no difference to cholesterol concentrations in most subjects. ${ }^{13} \mathrm{We}$ strongly recommend that all laboratories be prepared to determine non-fasting serum cholesterol concentration alone as a screening procedure. When the serum cholesterol concentration is less than $6.5 \mathrm{mmol} / 1$, measuring fasting triglycerides and $\mathrm{HDL}$ cholesterol identifies less than $2 \%$ of additional patients at increased risk of coronary heart disease. Restricting screening to cholesterol measurement is therefore the most cost effective approach. ${ }^{14}$ Triglyceride and HDL cholesterol measurement is necessary in patients with total cholesterol values of $>6.5 \mathrm{mmol} / 1$ to identify the lipoprotein abnormality. ${ }^{14}$ Whether nonfasting serum triglycerides and HDL cholesterol, with revised reference ranges, have any place in laboratory practice remains to be determined.

Most laboratories (75\%) provided services for HDL cholesterol measurement, four types of precipitation technique being used (fig 2). The most commonly used technique precipitated apolipoprotein B containing lipoproteins with phosphotungstate/magnesium, although method dependent bias compared with the reference technique of ultracentrifugation has been noted for this and other precipitation techniques. $^{13}$ There is no national EQAS scheme for this test. A recent survey from the United States of America has shown that although HDL cholesterol workload had increased by $284 \%$ over a five year period, this was not accompanied by improvements in performance. ${ }^{15}$ Further work on reference procedures, standardisation, and quality assurance is essential if HDL cholesterol analysis is to form part of coronary risk assessment based on consensus values. A good case can be made on economic and clinical grounds for limiting HDL cholesterol measurement to those patients in whom it will influence management, and our survey suggests that limited access policies are widely used. The higher degree of inaccuracy and imprecision inherent in HDL cholesterol measurement compared with total serum cholesterol estimation ${ }^{15}$ must be taken into account if HDL cholesterol concentrations are used for clinical decision making.

Few laboratories (14\%) measure apolipoprotein $\mathrm{AI}$ and $\mathrm{B}$ concentrations, although these investigations may also provide information on cardiovascular risk. ${ }^{16}{ }^{17}$ Problems with regard to reference methods, standardisation, calibration and quality assurance have been recognised and should be addressed before consensus values for clinical decision making can be considered. ${ }^{18}$ Similar reservations apply to the measurement of serum lipoprotein (a) concentrations $^{171920}$ for assessing coronary heart disease risk. Inclusion of these investigations in lipoprotein assessment would increase costs and more information from prospective as opposed to case-control studies is required before a clinical case can be made for apolipoprotein analysis to be widely available.

1 Lipid Research Clinics Program. The Lipid Research Clinics Coronary Primary Prevention Trial results. I. Reduction in the incidence of coronry heart disease. JAMA 1984;251:351-64.

2 Frick MH, Elo O, Haapa K, et al. Helsinki Heart Study primary prevention trial with gemfibrozil in middle-aged men with dyslipidemia. $N$ Engl $J$ Med 1987;317:1237-45.

3 Nikkilä EA, Viikiukoski P, Valle M, Frick MH. Prevention of progression of coronary atherosclerosis by treatment of hyperlipidaemia: a seven year prospective angiographic study. Br Med J 1984;289:220-3.

4 Blankenhorn DM, Nessim SA, Johnson RL, Sanmarco ME Azen SP, Cashin-Hemphill L. Beneficial effects Azen SP, Cashin-Hemphil L. Beneficial effects of (n) sclerosis and

5 Brown G, Albers JJ, Fisher LD, et al. Regression of coronry artery disease as a result of intensive lipid-lowering artery disease as a result of intensive lipid-lowering therapy in men with high levels

6 Consensus Conference. Lowering blood cholesterol to prevent heart disease. JAMA 1985;253:2080-6.

7 Study Group of the European Atherosclerosis Society. Strategies for prevention of coronary heart disease: policy statement of the European Atherosclerosis Society. Eur Heart $J$ 1987;8:77-88.

8 British Cardiac Society Working Group on Coronary Pre vention. Conclusions and recommendations. Br Heart 1987;57:188-9.

9 Shepherd J, Betteridge DJ, Durrington PN, et al. Strategies for reducing coronary heart disease and desirable limits for 
blood lipid concentrations: Guidelines of the British Hyperlipidaemia Association. BMJ 1987;295:1245-6.

10 Laboratory Standardization Panel. Current status of blood cholesterol measurement in clinical laboratories in the United States: A report from the Laboratory Standardization Panel of the National Cholesterol Education Program. Clin Chem 1988;34:193-201.

11 Laker MF, Reckless JPD, Betteridge DJ, et al. Facilities for the management of patients with lipid disorders in the the management of patients with lipid disorders in the
United Kingdom: results of the British Hyperlipidaemia United Kingdom: results of the British Hype

12 Boerma GJM, Jansen AP, Jansen RTP, Leiinse B, van Strik R. Minimizing interlaboratory variation in routine assays of serum cholesterol through the use of serum calibrators. Clin Chem 1986;32:943-7.

13 Durrington PN. Normal serum lipid and lipoprotein concentrations. Hyperlipidaemia Diagnosis and management. London: PG Wright, 1989:56-86.

14 Neil HAW, Mant D, Jones L, Morgan B, Mann JI. Lipid screening: Is it enough to measure total cholesterol concentration? BMJ 1990;301:584-7.

15 McMillan Ta, Warnick GR. Interlaboratory proficiency survey of cholesterol and high-density lipoprotein choles- terol measurement. Clin Chem 1988;34:1629-32.

16 Donohue RP, Orchard TJ, Stein EA, Kuller LH. Apolipoproteins AI, AII and B in young adults: Association with proteins AI, AII and B in young adults: Association with CHD risk factors. The B
Chron Dis 1986;39:823-30.

17 Durrington PN, Ishola M, Hunt L, Arrol S, Bhatnagar D. Apolipoproteins (a), AI and B and parental history in men with early onset ischaemic heart disease. Lancet 1988; i:1070-3.

18 Marcovina SM, Albers JJ. Standardization of the immunochemical determination of apolipoproteins A-I and B: A report on the International Federation of Clinical Chemisreport on the International Federation of Clinical ChemisB measurements (basis for future consensus), Vienna,

19 Seed M, Hoppichler F, Reaveley D, et al. Relation of serum lipoprotein(a) concentrations and apolipoprotein(a) phenotype to coronary heart disease in patients with familial hypercholesterolemia. $N$ Engl J Med 1990;322: 1494-9.

20 Mbewu A, Durrington PN. Lipoprotein (a): structure, properties and possible involvement in thrombogenesis and atherogenesis. Atherosclerosis 1990;85:1-14. 